\title{
The Missing Inferior Vena Cava with Acute on Chronic Undiagnosed DVT'S
}

\author{
Manov A*, Dhanabalsamy N, Gabbert B and Tola M \\ UNTHSC School of Medicine, USA
}

*Corresponding author: Andrey Manov, TCU and UNTHSC School of Medicine, 7016 Briercliff

Ct, Fort Worth, TX 76132, USA, Tel: 817-676-7605; Email: andrepenev@gmail.com

\section{Case Report}

Volume 3 Issue 1

Received Date: January 02, 2020

Published Date: February 04, 2020

DOI: $10.23880 /$ abca-16000113

\section{Introduction}

Deep venous thrombosis secondary to congenital anomalies involving Inferior Vena Cava (IVC) are extremely rare [1], they are seen in upto $5 \%$ of younger patient population with confirmed DVT [2]. Cautions should be taken to modify the risk factor that can cause hypercoagulable states. One such risk factor is IM testosterone which can cause polycythemia and stasis of blood thereby increasing the risk of clot formation, especially in patients who have hypercoagulable state. This is why testosterone is contraindicated in patients with hypercoagulable disorders. It is important to consider IVC anomalies in younger population who present with DVT to prevent life threatening pulmonary embolisms, acute limb ischemia and chronic leg ulcers in the future.

\section{Case Report}

A 45 year old caucasian man was evaluated in the emergency department with 3 month history he had 3-month history of mild swelling of the left lower extremity, heaviness and tingling which worsened 7-days prior to visit. Pt reported painful swelling in the groin which progressed to involve the entire left leg. It was associated with moderate to severe discomfort and tingling sensation. He denied any family history, recent trauma or travel, smoking, however, endorsed to have a similar swelling in his left groin three months ago along with gross hematuria while he was incarcerated. He was diagnosed with UTI during that episode, treated with antibiotics and his symptoms resolved.

During the current visit, he had left lower extremity $3+$ edema compared to the right lower extremity without neurovascular deficit, redness and varicose veins and pain on palpation over the deep vein system of the left lower extremity with areas of induration over the deep veins. Arterial pulsations on both lower extremities were preserved.
Patient was found to have polycythemia on CBC with HB18.4g/l and HCT-55\%.

Because of suspicion of deep venous thrombosis venous Doppler of the lower extremities was performed which was consistent with DVT of the entire deep venous system of the left leg extending all the way up into common iliac veins and Right Common Iliac vein. Clot was not completely hypoechoic suggesting that part of it to have been chronic with acute component as well-the ultrasonography diagnosis was acute on chronic Deep Venous Thrombosis/DVT/.

Patient also admitted using intramuscular testosterone cypionate $200 \mathrm{mg}$ weekly for last 4 months given in the Lo T-Center to improve his cognition.

Hypercoagulable workup was negative for Lupus anticoagulants, anticardiolipin antibodies-Beta-2 glycoprotein-1 antibodies, Factor $V$ Leiden mutation and pro thrombin 20210 mutations Lipoprotein/a/ level was normal. Also JAK 2 mutation was negative.

Interventional Radiology was consulted for catheter directed thrombolysis. A venogram by left popliteal approach was performed which showed extensive Left lower extremity acute DVT involving the left iliac veins and the deep venous system of the Left lower extremity distal to it with suspected chronic occlusion of the left common iliac vein. The Left common iliac vein could not be crossed despite exhaustive attempts.

This was followed by CT of the abdomen and pelvis with IV contrast was obtained which revealed chronic occlusion of congenitally malformed inferior vena cava/IVC/with extensive cavo-portal collaterals, acute short segment also Right common iliac acute DVT. The IVC at the Left common iliac vein confluence was diminutive. Interventional 
radiologist and vascular surgery recommended extensive ileocaval reconstruction but patient preferred conservative management.

Hematology was consulted and patient was started on anticoagulation with therapeutic dose heparin which was later used as a bridge to warfarin. Patient was advised not take IM testosterone for life. He improved during the hospital course and was discharged on therapeutic doses of Coumadin from the hospital.

\section{Discussion}

Congenital anomalies involving the IVC are rare and are under diagnosed [1]. Such anomalies occur in $0.3 \%$ of otherwise healthy individuals and in $0.6-2 \%$ of patients with other cardiovascular defects [2]. There is no data currently available regarding prophylactic anticoagulation for these patients. This particular patient population is susceptible to clot formation at baseline but their risk rises exponentially if they are exposed to factors that can contribute to hypercoagulable state $[3,4]$. Treatment is with anticoagulants/thrombolysis when appropriate. If not then vascular surgery is required to correct the underlying anomaly.

The other main problem with our patient was the use of IM Testosterone cypionate $200 \mathrm{mg}$ intramuscularly every week. The indication for prescribing Testosterone was improving his mental health which is not indication for using the product. Seconds-as per literature data Testosterone can cause DVT usually in the first 3-months after it's initiation $[5,6]$.

Third-usually those patients who develop clots on Testosterone have Factor $\mathrm{V}$ Leyden mutation or Antiphospholipid syndrome which our patient did not have [7]. This favors the argument that Testosterone on its own has thrombogenic properties, especially in patients with predisposing factors like our patient with congenital anomaly of IVC.

Fourth-we believe that the chronic clot was formed because of congenital anomaly of IVC and the acute, because of recent Testosterone use [8].
Fifth-this article underscores the need of thorough investigation and following the American endocrinology Society guidelines about the indications and contraindications of the Testosteron use.

\section{References}

1. Cho BC, Choi HJ, Kang SM, Chang J, Lee SM, et al. (2004) Congenital absence of inferior vena cava as a rare cause of pulmonary thromboembolism. Yonsei Med 45(5): 947-951.

2. Ackula H, Mosalpuria K, Tandra P (2016) Congenital agenesis of inferior vena cava as a rare cause of deep vein thrombosis: Case report and review of the literature. Case Reports in Internal Medicine 3(3): 74-77.

3. Puja SS, Ladia VM, Bajaj K (2014) Inferior Vena Cava Anomaly: A Risk for Deep Vein Thrombosis. North American journal of medical sciences 6(11): 601-603.

4. Gayer G, Luboshitz J, Hertz M, Zissin R, Thaler M, et al. (2003) Congenital Anomalies of the Inferior Vena Cava Revealed on CT in Patients with Deep Vein Thrombosis. American Journal of Roentgenology 180(3): 729-732.

5. Glueck CJ, Goldenberg N, Wang P (2018) Testosterone Therapy, Thrombophilia, Venous Thromboembolism, and Thrombotic Events. J Clin Med 8(1).

6. Glueck CJ, Goldenberg N, Budhani S, Lotner D, Abuchaibe C, et al. (2011) Thrombotic events after starting exogenous testosterone in men with previously undiagnosed familial thrombophilia. Transl Res 158(4): 225-234.

7. Glueck CJ, Prince M, Patel N, Patel J, Shah P, et al. (2016) Thrombophilia in 67 Patients With Thrombotic Events After Starting Testosterone Therapy. Clin Appl Thromb Hemost 22(6): 548-553.

8. Glueck CJ, Goldenberg N, Budhani S, Lotner D, Abuchaibe C, et al. (2011) Thrombotic events after starting exogenous testosterone in men with previously undiagnosed familial thrombophilia. Transl Res 158(4): $225-234$ 